\title{
Is there a role for humidified heated high-flow nasal cannula therapy in paediatric emergency departments?
}

\author{
Elliot Long, ${ }^{1,2,3}$ Franz E Babl, ${ }^{1,2,3}$ Trevor Duke ${ }^{2,3,4}$
}

\begin{abstract}
- Additional material is published online only. To view, please visit the journal online (http://dx.doi.org/10.1136/ emermed-2015-204914).

For numbered affiliations see end of article.
\end{abstract}

\section{Correspondence to} Dr Elliot Long, Department of Emergency Medicine, The Royal Children's Hospital, 50 Flemington Road, Parkville VIC 3052,Australia; Elliot.long@ rch.org.au

Received 16 April 2015 Revised 1 December 2015 Accepted 7 December 2015 Published Online First 4 January 2016
CrossMark

To cite: Long $\mathrm{E}$, Babl FE, Duke T. Emerg Med J 2016;33:386-389.

\begin{abstract}
Background Humidified heated high-flow nasal cannula (HFNC) therapy is a potentially useful form of non-invasive respiratory support for children with moderate respiratory distress and/or hypoxaemia. No prospective data support its use in the paediatric emergency department (ED). We introduced HFNC therapy into a paediatric ED and evaluated its use and failure rates.
\end{abstract}

Methods Prospective observational study of all patients presenting to the Royal Children's Hospital, Australia, who received HFNC therapy between April 2013 and September 2013 (one southern hemisphere winter season). We assessed demographics, indications, failure rate, predictors of failure and adverse events.

Results 71 patients commenced HFNC therapy in ED over the study period. The median age was 9 months. The most common indication was bronchiolitis (49/71; $69 \%$ ). Five (7\%) of the patients failed HFNC and were escalated to other forms of respiratory support in ED, four to nasal continuous positive airway pressure and one required intubation. A further 21 (32\%) failed HFNC therapy after intensive care unit (ICU) admission, giving a total failure of 28 (39\%). There were no serious adverse events in $\mathrm{ED}$, and one child with asthma developed air leak syndrome after transfer to the ICU. Conclusions HFNC therapy may have a role in the paediatric ED as an easily administered and well tolerated form of non-invasive respiratory support, but about one-third of patients required escalation to a higher level of respiratory support. Further studies should assess the safety profile of HFNC in larger series, and define the role of HFNC in key respiratory conditions compared with other possible interventions.

\section{INTRODUCTION}

Respiratory illness is the single most common reason for hospitalisation in children, and requirement for respiratory support is the most common indication for paediatric intensive care unit (PICU) admission overall. ${ }^{1}$ Therefore, there is considerable potential benefit from a non-invasive form of respiratory support that is easy to apply and care for, and is tolerable to patients, such as high-flow nasal cannula therapy (HFNC therapy). The prototypical illness where this might apply is in infants with bronchiolitis, where medical therapies have not been shown to be effective in reducing symptoms or altering the disease course. ${ }^{2} 3$

HFNC therapy was first described as a noninvasive method for delivering positive airway pressure in preterm neonates. ${ }^{4}$ It has subsequently been applied to a broader patient group with moderate to severe respiratory distress or hypoxaemia not

\section{Key messages}

What is already known on this subject?

- High-flow nasal cannula (HFNC) therapy provides some respiratory support in the form of positive airway pressure, gas conditioning and titratable fraction of inspired oxygen.

- HFNC therapy is associated with reduced intubation rates for acute respiratory failure in children in the paediatric intensive care unit.

\section{What might this study add?}

- In this prospective study of 71 children receiving high-flow nasal cannula (HFNC) in a paediatric ED, approximately one-third commenced on HFNC received escalation of respiratory support in the ED or during hospitalisation.

relieved by standard oxygen therapy. Possible indications include children with bronchiolitis, pneumonia, congestive cardiac failure, neuromuscular disease, apnoea of prematurity and as respiratory support following extubation from mechanical ventilation or weaning from other forms of noninvasive respiratory support. ${ }^{5}$ HFNC is thought to be of benefit in acute respiratory failure by decreasing upper airway resistance, washing out anatomical dead space, reducing the metabolic cost of gas conditioning and delivering variable and unmeasured positive airway pressure. $^{6}{ }^{7}$ It also allows the administration of variable fraction of inspired oxygen $\left(\mathrm{FiO}_{2}\right)$ by minimising entrainment of room air. Flow rates described as constituting high flow are variable, ranging from 1 to $2 \mathrm{~L} / \mathrm{kg} / \mathrm{min}$, with some evidence that higher flow rates deliver higher positive airway pressure, ${ }^{8} 9$ and that air leak around the nares and mouth opening significantly affect delivered pressure. ${ }^{10}$ There is currently limited evidence to support the efficacy and safety of HFNC for its use as a form of respiratory support in the treatment of the variety of indications for which it is currently used. ${ }^{7}$ As with any form of positive airway pressure, HFNC may result in the development of air leak syndromes and may decrease venous return and thus cardiac output. ${ }^{11}$ The major perceived benefits of HFNC over other forms of non-invasive respiratory support are its ease of application and level of nursing care required, and its patient tolerability without requiring sedation in the majority of patients compared with nasal continuous positive airway pressure (CPAP). ${ }^{12}$ The use of intermediate flow rates 
(below $1 \mathrm{~L} / \mathrm{kg} / \mathrm{min}$ ), and the delivery of humidified oxygen at conventional (low) flow rates or high flow rates through face masks do not provide any positive airway pressure and therefore do not constitute HFNC therapy in this context.

Randomised controlled trials in preterm infants indicate that HFNC therapy has similar efficacy to nasal CPAP as an initial form of respiratory support ${ }^{13}$ and as postextubation respiratory support. ${ }^{14}$ One multicentred prospective randomised trial comparing HFNC, bilevel positive airway pressure (BiPAP) and nonrebreather face mask oxygen for acute hypoxaemic respiratory failure in adults found no significant difference in subsequent intubation rate. ${ }^{15}$ There have been no randomised trials of HFNC therapy in any other setting. Observational studies suggest that HFNC therapy, when introduced to PICUs, is associated with a reduction in intubation rate, that it is safe, and associated with failure rates of around $25 \%-30 \% .^{5} 16$ Emergency department (ED) data on the use of HFNC therapy are limited. Retrospective observational data from before and after the introduction of HFNC therapy in the paediatric ED did not show a reduction in intubation rate for children with acute respiratory failure, however the ICU admission rate was increased. ${ }^{17}$

We introduced HFNC therapy in the paediatric ED under hospital-wide guidelines for its use over a 6-month trial phase. As this has not been previously described, we documented the patient demographics, indications, failure rate and adverse events prospectively over this introductory period. We assessed if patients who failed HFNC could be predicted based on response to therapy within $2 \mathrm{~h}$ of initiation.

\section{METHODS}

We undertook a prospective evaluation of patients receiving HFNC therapy during the first 6 months after its introduction in the paediatric ED of the Royal Children's Hospital ( $\mathrm{RCH})$, Melbourne, Australia. The RCH ED has over 85000 presentations annually, approximately 1400 diagnosed with bronchiolitis, of whom $580(40 \%)$ are admitted to hospital and 50 (3.5\%) to ICU. The study was approved by the hospital's Human Research and Ethics Committee.

Following the introduction of HFNC therapy in the ICU at $\mathrm{RCH}$, a guideline for standardised set-up and delivery was developed (see online supplementary appendix 1$).^{5}$ The guideline refers to a single proprietary HFNC delivery system that is used in our institution, though multiple other delivery systems exist. ${ }^{18}$ Equipment and training were provided to ED staff prior to introduction of HFNC. The flow rate used during the study period was $2 \mathrm{~L} / \mathrm{kg} / \mathrm{min}$ for the first $10 \mathrm{~kg}$, then $0.5 \mathrm{~L} / \mathrm{kg} / \mathrm{min}$ for every kilogram thereafter. Indications for use of HFNC were broadly defined as moderate to severe respiratory distress where increased work of breathing or hypoxaemia was not relieved by standard oxygen therapy.

Study patients were identified prospectively by their treating clinician, who completed a clinical report form at the time of patient encounter (see online supplementary appendix 2). Patients of any age who were started on HFNC therapy in ED were included. There were no exclusions. Patients age, weight, diagnosis, comorbidities, vital signs before starting HFNC (HR, $\mathrm{RR}$ and oxygen saturations $\left(\mathrm{SpO}_{2}\right)$ ), flow rate and fraction of inspired oxygen $\left(\mathrm{FiO}_{2}\right)$ on initiation, vital signs and $\mathrm{FiO}_{2} 2 \mathrm{~h}$ after initiation, sedation requirement, outcome and adverse events were recorded by the ED treating clinician. Missing data points were obtained from the patient's records or through direct contact with the treating clinician. The ED electronic record was searched weekly to ensure that all patients started on
HFNC were captured, and the ICU admission log was crossreferenced to ensure that no patients were missed (at the time of the study all children admitted on HFNC were managed in ICU).

Failure of HFNC therapy was defined as escalation of respiratory support to another form of non-invasive ventilation (nasal CPAP) or BiPAP or invasive ventilation within $24 \mathrm{~h}$ of initiation of HFNC therapy. Normalisation of vital signs was defined according to hospital-wide medical emergency team criteria. ${ }^{19}$

Data were entered into a Microsoft Excel 2010 (Microsoft, Redmond, Washington, USA) database. Descriptive statistics were used for key proportions.

\section{RESULTS}

Over the 6-month study period, 71 patients were started on HFNC therapy. Sixty-nine (97\%) of the patients received flow rates according to the guidelines. The median age was 9 months, and the most common diagnosis was bronchiolitis (table 1). Comorbidities were uncommon.

Patients receiving HFNC were transferred to ICU for ongoing management (table 2). Failure of HFNC therapy in ED occurred in five patients (7\%). Four were escalated to nasal CPAP in ED, and one was intubated in ED. Following admission to PICU, 16 (23\%) patients were escalated to nasal CPAP and seven $(10 \%)$ intubated. Overall 28 children required escalation of respiratory support, giving a failure rate of $39 \%$.

Twenty-four patients (34\%) were treated with chloral hydrate for sedation following the initiation of HFNC therapy.

There were no adverse events in ED except three patients developed abdominal distension. After transfer to ICU one patient on HFNC developed air leak syndrome. This occurred in a 4-year-old girl with severe asthma treated with HFNC at a flow rate of $40 \mathrm{~L} / \mathrm{min}$ for $10 \mathrm{~h}$. Bilateral pneumothoraces, pneumomediastinum, pneumopericardium and subcutaneous emphysema developed, requiring bilateral chest drain insertion and intubation.

\section{DISCUSSION}

In this prospective study of HFNC as a method for providing respiratory support we found it to be well tolerated and easily administered. Overall $39 \%$ of patients failed HFNC therapy.

Two retrospective studies have examined the use of HFNC in the paediatric ED. The first compared intubation rates in ED before and after the introduction of HFNC as a form of respiratory support. ${ }^{17}$ The authors found a significant reduction in ED intubation rates after the introduction of HFNC therapy. Total intubation rates over the study period (ED and PICU combined) did not change, and the reduction in intubation rates in ED was associated with a concomitant increase in intubation rates during ongoing care in PICU. Though only $7 \%$ patients in our study failed HFNC in ED, 28\% went on to fail HFNC therapy during their subsequent hospitalisation. PICU admission rates have been variably affected after the introduction of HFNC therapy in EDs or on wards. ${ }^{17}{ }^{20}$ Clear predictors of failure of HFNC and wards trained and equipped to manage children successfully initiated on HFNC seem to be critical in reducing PICU admission rates following the introduction of HFNC. Observational studies performed in PICU support the notion that HFNC therapy reduces intubation rates for acute respiratory compromise, ${ }^{16}{ }^{21}$ though to date no randomised comparison of HFNC with other forms of respiratory support have been performed outside neonatal ICU. The increase in PICU admission rates should HFNC be introduced in ED raises the issue of disposition of patients on HFNC therapy. Should 
Table 1 Demographic information, indication and initial vital signs for patients treated with HFNC therapy in the ED

\begin{tabular}{|c|c|c|c|}
\hline & All patients $(n=71)$ & Successfully initiated on HFNC $(n=43)$ & Failed HFNC $(n=28)$ \\
\hline Age (months) (median, IQR) & $9(3-18)$ & $12(7-20)$ & $8(4-15)$ \\
\hline Male n (\%) & $41(58)$ & $23(53)$ & $18(64)$ \\
\hline \multicolumn{4}{|l|}{ Comorbidity n (\%) } \\
\hline Nil & $62(87)$ & $37(86)$ & $25(89)$ \\
\hline Chronic lung disease & $4(6)$ & $3(7)$ & $1(4)$ \\
\hline Congenital heart disease & $3(4)$ & $3(7)$ & $0(0)$ \\
\hline Cerebral palsy & $2(3)$ & $0(0)$ & $2(7)$ \\
\hline \multicolumn{4}{|l|}{ Indication for HFNC n (\%) } \\
\hline Bronchiolitis & $49(69)$ & $28(65)$ & $21(75)$ \\
\hline Acute lower respiratory tract infection & $17(24)$ & $11(26)$ & $6(21)$ \\
\hline Asthma & $2(3)$ & $1(2)$ & $1(3)$ \\
\hline Sepsis & $1(1)$ & $1(2)$ & $0(0)$ \\
\hline Apnoea & $1(1)$ & $1(2)$ & $0(0)$ \\
\hline Cardiac disease & $1(1)$ & $1(2)$ & $0(0)$ \\
\hline \multicolumn{4}{|l|}{ Initial vital signs } \\
\hline HR; median (IQR) & $167(152-182)$ & $164(153-179)$ & $172(150-185)$ \\
\hline RR; median (IQR) & $63(54-70)$ & $60(52-65)$ & $68(56-76)$ \\
\hline Temperature $\left({ }^{\circ} \mathrm{C}\right)$; median (IQR) & $37.5(37.2-37.9)$ & $37.5(37.3-37.9)$ & $37.5(37.2-39.9)$ \\
\hline Oxygen saturation (\%); median (IQR) & 97 (96-99) & $97(96-98)$ & 97 (96-99) \\
\hline \multicolumn{4}{|l|}{ Vital signs $2 \mathrm{~h}$ after initiation of HFNC } \\
\hline HR; median (IQR) & $151(139-167))$ & $144(136-159)$ & $157(151-170)$ \\
\hline RR; median (IQR) & $58(52-65)$ & $46(41-50)$ & $61(55-67)$ \\
\hline Temperature $\left({ }^{\circ} \mathrm{C}\right)$; median (IQR) & $37.3(37.1-37.8)$ & $37.3(37.0-37.8)$ & $37.4(37.1-37.8)$ \\
\hline Oxygen saturation (\%); median (IQR) & 96 (94-99) & 97 (95-98) & $96(94-98)$ \\
\hline
\end{tabular}

HFNC, high-flow nasal cannula.

hospital wards not be trained, equipped and supported to deliver HFNC, its introduction to ED may increase PICU admission rates. At the time our study took place, all patients initiated on HFNC therapy in ED were transferred to PICU for ongoing care. With limited evidence for safety outside of critical care areas $^{22}$ ongoing HFNC therapy has subsequently been trialled successfully on our hospital wards.

The second retrospective study of HFNC therapy in the paediatric ED looked at predictors of failure of HFNC therapy. ${ }^{23}$ The authors found that tachypnoea ( $>90$ th percentile for age), an initial $\mathrm{pCO}_{2}>50 \mathrm{~mm} \mathrm{Hg}$ and an initial venous $\mathrm{pH}$ of $<7.30$ were associated with failure of HFNC therapy. One or more of these predictors were present in 23 of the 28 patients $(82 \%)$ who failed HFNC overall in our study. Twenty-two of

Table 2 Disposition and highest level of respiratory support during hospitalisation in patients initiated on HFNC therapy in the ED

\begin{tabular}{lc}
\hline Outcome following initiation of HFNC $n(\%)$ & \\
Transferred to PICU on HFNC & $64(90)$ \\
Escalation to nCPAP in ED & $3(4)$ \\
Escalation to intubation in ED & $1(1)$ \\
HFNC not tolerated & $2(3)$ \\
Weaned to low-flow oxygen in ED & $1(1)$ \\
Highest level of respiratory support during admission n (\%) & \\
HFNC & $43(61)$ \\
nCPAP & $20(28)$ \\
ETT & $8(11)$
\end{tabular}

ETT, endotracheal tube; HFNC, high-flow nasal cannula; nCPAP, nasal continuous positive airway pressure; PICU, paediatric intensive care unit. the 43 patients (51\%) who did not fail HFNC therapy had continued abnormalities of one or more of these parameters at $2 \mathrm{~h}$. Additional studies in the PICU environment have found lower initial RR, high initial $\mathrm{pCO}_{2}$ and failure of $\mathrm{RR}$ to improve/ saturations to improve with HFNC to be predictive of failure. ${ }^{24}$

The overall failure rate for HFNC therapy in the ICU has been reported as around 30\%, with 5\%-10\% requiring escalation to invasive ventilation. ${ }^{5}{ }^{16}$ In our study, $7 \%$ patients failed HFNC therapy in ED, and $32 \%$ subsequently failed HFNC therapy in PICU. Overall the need for endotracheal intubation was $11 \%$. A $39 \%$ total failure rate is similar overall to those reported from the ICU setting.

The adverse event rate in our study was low, with no serious adverse events occurring in ED. During ongoing care in PICU, one patient developed an air leak syndrome. It should be noted that this patient was being treated for severe asthma, where air leak syndrome has been reported even in patients receiving no respiratory support. ${ }^{25}$ Additionally, though air leak syndrome has been reported at low-flow rates, ${ }^{26}$ the flow rate being delivered during this adverse event was higher than that recommended in our current clinical guideline. In this small, non-comparative, single centred observational study, it is difficult to draw conclusions regarding the relative safety of HFNC compared with other forms of non-invasive respiratory support. Nevertheless, an index of suspicion should exist for the potential development of air leak syndromes in patients treated with HFNC.

\section{Limitations}

Though every effort was made to identify all patients initiated on HFNC during the study period, there may have been some patients missed who rapidly deteriorated to invasive respiratory 
support after a short trial of HFNC. Our study was observational and non-comparative in nature and therefore could not be used to comment on the efficacy of HFNC therapy or the flow rate that should optimally be used.

\section{CONCLUSION}

HFNC oxygen therapy appears to be a feasible method for delivering respiratory support, which can be initiated in the paediatric ED. Ongoing and expanded use of this modality of respiratory support will require more extensive safety data and efficacy should be assessed in an interventional study comparing HFNC with other forms of respiratory support. A multicentre study comparing HFNC and low-flow nasal prong therapy has commenced in Australia and New Zealand.

\author{
Author affiliations \\ ${ }^{1}$ Department of Emergency Medicine, The Royal Children's Hospital, Parkville, \\ Victoria, Australia \\ ${ }^{2}$ Murdoch Children's Research Institute, Parkville, Victoria, Australia \\ ${ }^{3}$ Department of Paediatrics, Faculty of Medicine, Dentistry, and Health Sciences, \\ University of Melbourne, Parkville, Victoria, Australia \\ ${ }^{4}$ Paediatric Intensive Care Unit, The Royal Children's Hospital, Parkville, Victoria, \\ Australia
}

Acknowledgements The study investigators would like to thank clinical staff for completing the clinical report forms, the Royal Children's Hospital ED educators for providing ongoing high-flow nasal cannula (HFNC) training and paediatric intensive care unit outreach staff for supporting the use of HFNC outside of the intensive care unit.

Contributors EL designed the study, collected the data and wrote the manuscript. FEB submitted the ethics application and revised the manuscript. TD wrote the high-flow guideline included as an appendix and revised the manuscript.

Funding This study was supported by a National Health and Medical Research Council Centre of Research Excellence Grant for Paediatric Emergency Medicine (GNT1058560), Canberra, Australian Capital Territory, Australia and the Victorian Government's Infrastructure Support Program, Melbourne, Australia.

Competing interests None declared.

Ethics approval Human Research in Ethics Committee at the Royal Children's Hospital Melbourne (approval number: 32214 A).

Provenance and peer review Not commissioned; externally peer reviewed.

\section{REFERENCES}

1 Alexander J, Tregean S, Slater A. Report of the Australian and New Zealand Paediatric Intensive Care Registry 2011. Australian and New Zealand Intensive Care Society, 2011.

2 Ralston SL, Lieberthal AS, Meissner HC, et al. Clinical practice guideline: the diagnosis, management, and prevention of bronchiolitis. Pediatrics 2014;134: e1474-502.

3 Everard ML, Hind D, Ugonna K, et al. SABRE: a multicentre randomised control trial of nebulised hypertonic saline in infants hospitalised with acute bronchiolitis. Thorax 2014;69:1105-12.
4 Locke RG, Wolfson MR, Shaffer TH, et al. Inadvertent administration of positive end-distending pressure during nasal cannula flow. Pediatrics 1993;91:135-8.

5 ten Brink F, Duke T, Evans J. High-flow nasal prong oxygen therapy or nasopharyngeal continuous positive airway pressure for children with moderate-to-severe respiratory distress?*. Pediatr Crit Care Med 2013;14:e326-31.

6 Lee JH, Rehder $\mathrm{KJ}$, Williford L, et al. Use of high flow nasal cannula in critically ill infants, children, and adults: a critical review of the literature. Intensive Care Med 2013;39:247-57.

7 Dysart K, Miller TL, Wolfson MR, et al. Research in high flow therapy: mechanisms of action. Respir Med 2009;103:1400-5.

8 Milési C, Boubal M, Jacquot A, et al. High-flow nasal cannula: recommendations for daily practice in pediatrics. Ann Intensive Care 2014;4:29.

9 Hough JL, Pham TM, Schibler A. Physiologic effect of high-flow nasal cannula in infants with bronchiolitis. Pediatr Crit Care Med 2014;15:e214-9.

10 Hasan RA, Habib RH. Effects of flow rate and airleak at the nares and mouth opening on positive distending pressure delivery using commercially available high-flow nasal cannula systems: a lung model study. Pediatr Crit Care Med 2011;12:e29-33.

11 Bronicki RA, Anas NG. Cardiopulmonary interaction. Pediatr Crit Care Med 2009;10:313-22.

12 Hough JL, Shearman AD, Jardine LA, et al. Humidified high flow nasal cannulae: current practice in Australasian nurseries, a survey. J Paediatr Child Health 2012;48:106-13.

13 Yoder BA, Stoddard RA, Li M, et al. Heated, humidified high-flow nasal cannula versus nasal CPAP for respiratory support in neonates. Pediatrics 2013;131: e1482-90.

14 Manley BJ, Owen LS, Doyle LW, et al. High-flow nasal cannulae in very preterm infants after extubation. N Engl J Med 2013;369:1425-33.

15 Frat JP, Thille AW, Mercat A, et al. High-flow oxygen through nasal cannula in acute hypoxemic respiratory failure. N Engl J Med 2015;372:2185-96.

16 Schibler A, Pham TM, Dunster KR, et al. Reduced intubation rates for infants after introduction of high-flow nasal prong oxygen delivery. Intensive Care Med 2011:37:847-52.

17 Wing R, James C, Maranda LS, et al. Use of high-flow nasal cannula support in the emergency department reduces the need for intubation in pediatric acute respiratory insufficiency. Pediatr Emerg Care 2012;28:1117-23.

18 Ward JJ. High-flow oxygen administration by nasal cannula for adult and perinatal patients. Respir Care 2013;58:98-122.

19 Tibballs J, Kinney S. Reduction of hospital mortality and of preventable cardiac arrest and death on introduction of a pediatric medical emergency team. Pediatr Crit Care Med 2009;10:306-12.

20 Mayfield S, Bogossian F, O'Malley L, et al. High-flow nasal cannula oxygen therapy for infants with bronchiolitis: pilot study. J Paediatr Child Health 2014;50:373-8.

21 McKiernan C, Chua LC, Visintainer PF, et al. High flow nasal cannulae therapy in infants with bronchiolitis. J Pediatr 2010;156:634-8.

22 Bressan S, Balzani M, Krauss B, et al. High-flow nasal cannula oxygen for bronchiolitis in a pediatric ward: a pilot study. Eur J Pediatr 2013;172:1649-56.

23 Kelly GS, Simon HK, Sturm JJ. High-flow nasal cannula use in children with respiratory distress in the emergency department: predicting the need for subsequent intubation. Pediatr Emerg Care 2013;29:888-92.

24 Abboud PA, Roth PJ, Skiles $\mathrm{CL}$, et al. Predictors of failure in infants with viral bronchiolitis treated with high-flow, high-humidity nasal cannula therapy*. Pediatr Crit Care Med 2012;13:e343-9.

25 Leigh-Smith S, Christey G. Tension pneumothorax in asthma. Resuscitation 2006:69:525-7.

26 Hegde S, Prodhan P. Serious air leak syndrome complicating high-flow nasal cannula therapy: a report of 3 cases. Pediatrics 2013;131:e939-44. 